\title{
Precipitation estimation over radar gap areas based on satellite and adjacent radar observations
}

\author{
Y.-R. Lee ${ }^{1}$, D.-B. Shin ${ }^{1}$, J.-H. Kim ${ }^{1}$, and H.-S. Park ${ }^{2}$ \\ ${ }^{1}$ Department of Atmospheric Sciences, Yonsei University, Seoul, South Korea \\ ${ }^{2}$ Radar Analysis Division, Weather Radar Center, KMA, Seoul, South Korea \\ Correspondence to: D.-B. Shin (dbshin@yonsei.ac.kr)
}

Received: 24 May 2014 - Published in Atmos. Meas. Tech. Discuss.: 23 June 2014

Revised: 31 December 2014 - Accepted: 19 January 2015 - Published: 11 February 2015

\begin{abstract}
Continuous rainfall measurements from groundbased radars are crucial for monitoring and forecasting heavy rainfall-related events such as floods and landslides. However, complete coverage by ground-based radars is often hampered by terrain blockage and beam-related errors. In this study, we presented a method to fill the radar gap using surrounding radar-estimated precipitation and observations from a geostationary satellite. The method first estimated the precipitation over radar gap areas using data from the Communication, Ocean, and Meteorological Satellite (COMS); the first geostationary satellite of Korea. The initial precipitation estimation from COMS was based on the rain ratebrightness temperature relationships of a priori databases. The databases were built with temporally and spatially collocated brightness temperatures at four channels $(3.7,6.7,10.8$, and $12 \mu \mathrm{m})$ and Jindo $\left(126.3^{\circ} \mathrm{E}, 34.5^{\circ} \mathrm{N}\right)$ radar rain rate observations. The databases were updated with collocated data sets in a timespan of approximately one hour prior to the designated retrieval. Then, bias correction based on an ensemble bias factor field (Tesfagiorgis et al., 2011b) from radar precipitation was applied to the estimated precipitation field. Over the radar gap areas, this method finally merged the biascorrected satellite precipitation with the radar precipitation obtained by interpolating the adjacent radar observation data. The merging was based on optimal weights determined from the root-mean-square error (RMSE) with the reference sensor observation or equal weights in the absence of reference data. This method was tested for major precipitation events during the summer of 2011 with assumed radar gap areas. The results suggested that successful merging appears to be closely related to the quality of the satellite precipitation estimates.
\end{abstract}

\section{Introduction}

The need for continuous monitoring and accurate measurement of precipitation is critical for the efficiency of severe weather and hazard predictions, such as that of flash floods, landslides, and extreme rainfall forecasts. Precipitation is conventionally estimated from ground-based radar by observing the backscattered reflectivity from raindrops (Austin, 1987). The precipitation estimates obtained from radars are quite beneficial to weather forecasters and meteorologists, because radar-estimated rain rate information is available in high spatial and temporal resolutions. However, there may be observational gap areas caused by terrain blockage and beam breakdown. In particular, radar estimates can be significantly absent over mountainous regions where heavy rainfalls can occur. Assuring the continuous measurement of precipitation highly varying in space and time creates the need for merging available observations over the gap areas.

Precipitation estimates from geostationary satellites may be considered to mitigate the discontinuity in radar observations because the satellites provide large observational coverage at high spatial and temporal resolutions. Despite the advantage of frequent observation from geostationary satellites, the primary infrared(IR)-based sensors onboard the satellites only detect cloud-top brightness temperatures that are indirectly related to the physical process of surface precipitation (Kidd et al., 2003). The ground-based radar, which is an active microwave sensor, can estimate precipitation more accurately than IR-based precipitation estimation, because microwave sensors can detect radiation directly from water droplets or ice particles within clouds. Therefore, better precipitation estimates over radar gap areas may be attained by 
Table 1. List of precipitation cases for the precipitation estimations from the geostationary satellite and construction of a priori databases.

\begin{tabular}{|c|c|c|c|}
\hline \multirow[t]{2}{*}{ Precipitation case } & \multirow{2}{*}{$\begin{array}{l}\text { Selected date and time } \\
\text { for precipitation } \\
\text { retrieval } \\
\text { (YYMMDDHHMM) }\end{array}$} & \multicolumn{2}{|c|}{$\begin{array}{l}\text { Date and time of radar and satellite } \\
\text { observations for a priori databases } \\
\text { (YYMMDDHHMM) }\end{array}$} \\
\hline & & Radar & Satellite \\
\hline \multirow{5}{*}{1} & \multirow{5}{*}{1107091400} & 1107091340 & 1107091345 \\
\hline & & 1107091330 & 1107091330 \\
\hline & & 1107091310 & 1107091315 \\
\hline & & 1107091300 & 1107091300 \\
\hline & & 1107091230 & 1107091230 \\
\hline \multirow{5}{*}{2} & \multirow{5}{*}{1107092100} & 1107092040 & 1107092045 \\
\hline & & 1107092000 & 1107092000 \\
\hline & & 1107091940 & 1107091945 \\
\hline & & 1107091930 & 1107091930 \\
\hline & & 1107091900 & 1107091900 \\
\hline \multirow{5}{*}{3} & \multirow{5}{*}{1107121200} & 1107121140 & 1107121145 \\
\hline & & 1107121100 & 1107121100 \\
\hline & & 1107121040 & 1107121045 \\
\hline & & 1107121030 & 1107121030 \\
\hline & & 1107121000 & 1107121000 \\
\hline \multirow{5}{*}{4} & \multirow{5}{*}{1108072300} & 1108072240 & 1108072245 \\
\hline & & 1108072230 & 1108072230 \\
\hline & & 1108072210 & 1108072215 \\
\hline & & 1108072200 & 1108072200 \\
\hline & & 1108072130 & 1108072130 \\
\hline
\end{tabular}

utilizing the adjacent radar estimated precipitation and satellite estimations.

Some researchers have attempted to produce precipitation information using multi-sensor estimated precipitation. Kondragunta et al. (2005) integrated the satellite precipitation estimates after local bias correction with radar and rain gauge data in order to fill missing radar observations. Mahani and Khanbilvardi (2009) generated multi-sensor precipitation products by merging satellite precipitation estimates from the Hydro-Estimator (HE) algorithm (Scofield and Kuligowski, 2003) with ground-based radar observations in order to improve satellite-based precipitation retrieval. Their results showed that merged precipitation tends to be more accurate than satellite only driven precipitation over radar gap areas. A similar approach was performed by Tesfagiorgis et al. (2011a). The approach applied bias correction to the satellite precipitation estimates obtained from the HE algorithm and proposed a successive correction method in order to merge the satellite precipitation estimates with the radar observation.

In this study, we developed a method of precipitation estimation over radar gap areas based on the merging of geostationary satellite and radar observations currently operated by the Korea Meteorological Administration (KMA). We used a geostationary satellite called the Communication, Ocean, and Meteorological Satellite (COMS) which was launched in June 2010. COMS has four IR channels and one visible channel with band centers located at 3.7, 6.7, 10.8, 12.0, and $0.67 \mu \mathrm{m}$, respectively (Choi et al., 2007). COMS provides the brightness temperature $\left(T_{\mathrm{b}}\right)$ from IR channels every $15 \mathrm{~min}$, which has a $4 \mathrm{~km}$ spatial resolution over the Korean Peninsula. Precipitation estimates using IR $T_{\mathrm{b}}$ from geostationary satellites have been presented in previous studies (e.g., Vicente et al., 1998; Scofield and Kuligowski, 2003). In this study, we independently estimated precipitation from COMS based on a reference of radar observations. The methodology for estimating the precipitation from a geostationary satellite and the merging technique of radar and satellite precipitation estimates are described in Sect. 2. This method was tested with four precipitation cases during the summer of 2011 (Table 1). The results of the merged precipitations are presented in Sect. 3. A summary of our study is presented in Sect. 4.

\section{Methodology}

The methodology described in this study was designed to create a merged radar and satellite estimated precipitation field over radar gap areas. Figure 1 shows a schematic representation of merging the radar and satellite precipitation estimates. The methodology consisted of two parts. The first part (Block 1) is related to the retrieval of 


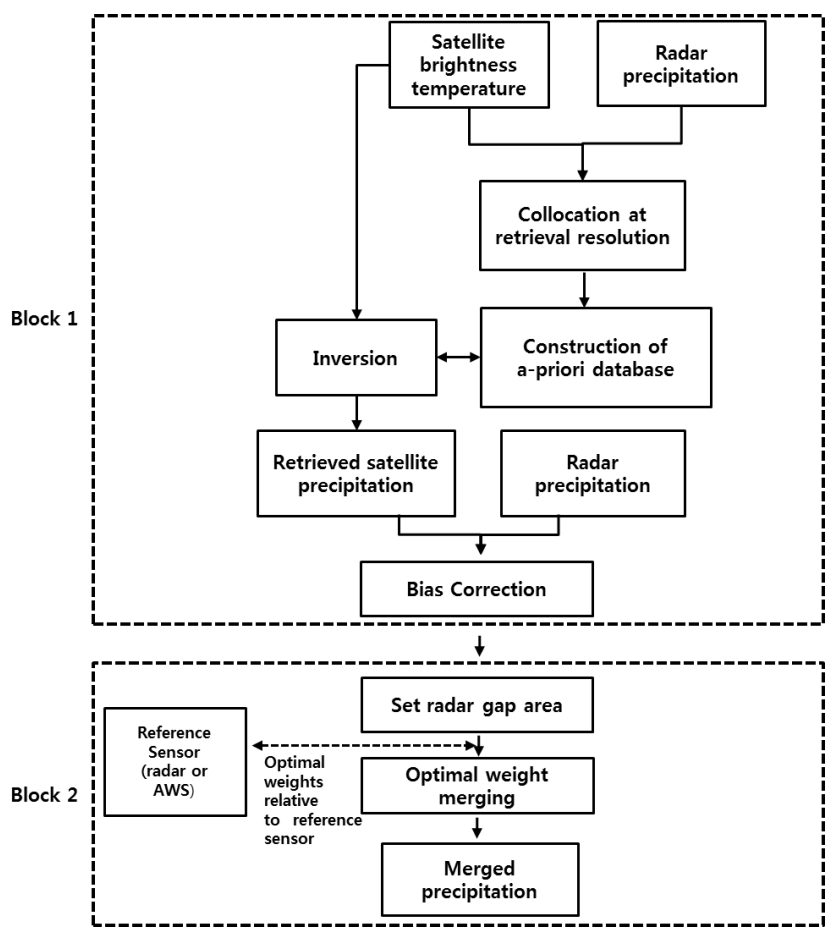

Figure 1. A flow diagram to estimate the precipitation over a radar gap area by merging the radar and satellite data.

satellite precipitation based on an a priori database. The other part (Block 2) illustrates the procedure for merging satellite precipitation estimates and adjacent radar observations with optimally determined weights based on reference measurements. The details of the methodology are discussed in the following sub-sections. Among the 11 Doppler weather radars currently operated by KMA over the Korean Peninsula, the radar at Jindo, in the west part of the Korean Peninsula (Fig. 2), was used in this study. The Jindo radar uses the S-band typical for weather radars with an observation range of $240 \mathrm{~km}$ radius. It primarily monitors precipitation and storms approaching from the west of the Korean Peninsula.

\subsection{Satellite precipitation estimation}

\subsubsection{The databases}

Precipitation estimations from satellites in this study were principally based on the relationships between the rain rate and the satellite-observed $T_{\mathrm{b}}$ characterized by a priori databases. In the construction of the a priori database, the radar-estimated rain rates were collected to ensure consistency with the radar. Then, the brightness temperatures at the four channels $(3.7,6.7,10.8$, and $12 \mu \mathrm{m})$ of COMS were collocated temporally and spatially with the radar rain rate observations in the databases. Downscaling of the satellite data to $1 \mathrm{~km}$ was performed in order to collocate the

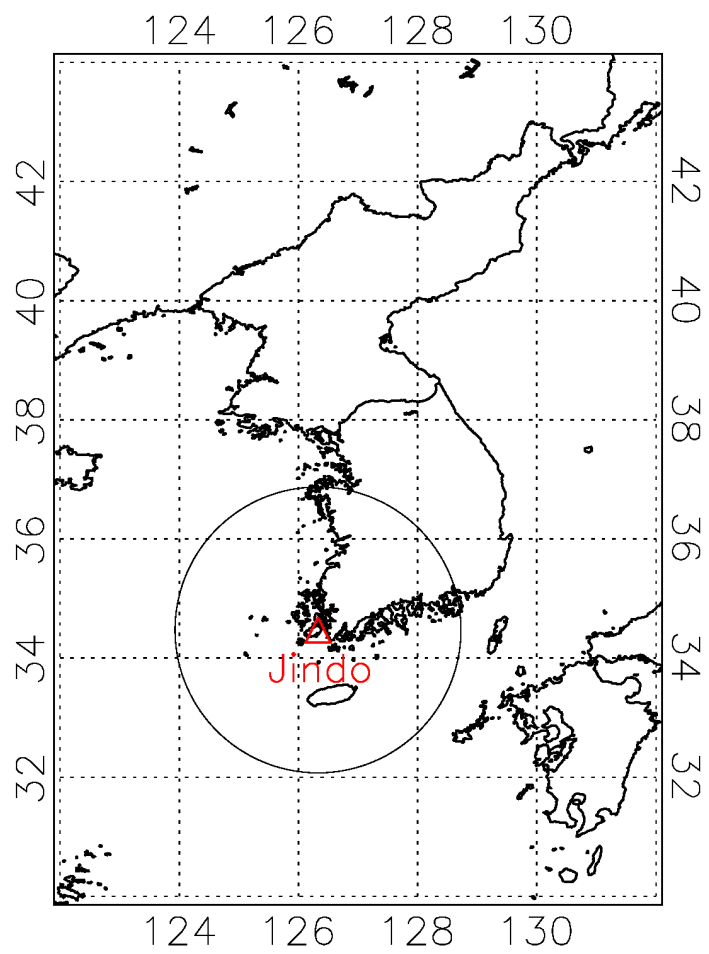

Figure 2. The location of the Jindo radar (red triangle) and its observational coverage (black circle), which indicates the study area.

radar and satellite data, because of the difference between the radar $(1 \times 1 \mathrm{~km})$ and satellite $(4 \times 4 \mathrm{~km})$ spatial resolutions. A satellite pixel was split into 4 pixels in both the longitudinal and latitudinal directions. Then, the collocated satellite brightness temperature $\left(T_{\mathrm{b}_{\mathrm{col}}}\right)$ was computed as follows:

$T_{\mathrm{b}_{\mathrm{col}}}=\frac{\sum_{i=1}^{n} \mathrm{~W}_{i} \cdot T_{\mathrm{b}_{\text {org }}}}{\sum_{i=1}^{n} \mathrm{~W}_{i}}$

and

$\mathrm{W}_{i}=\exp \left(\frac{-r^{2}}{2 \cdot \text { res }_{\text {radar }}}\right)$,

where $T_{\mathrm{b}_{\text {org }}}$, res radar $_{\text {rar }}$, and $r$ indicate the $T_{\mathrm{b}}$ at the original resolution $(4 \times 4 \mathrm{~km})$, the radar spatial resolution $(1 \mathrm{~km})$, and the distance between the satellite sub-pixel and radar pixel at location $i$. This collocation method applies to the $T_{\mathrm{b}}$ at each of the four channels. After collocation, the radar rain rate and corresponding satellite $T_{\mathrm{b}}$ was collected over the radar observational area for each of the selected precipitation cases. The databases also included non-raining areas of the $T_{\mathrm{b}}$ in order to discriminate non-raining areas.

In order to include the database elements reasonably close to the physical properties of a target scene, the database was supposed to be continuously updated in a time span of approximately 1.5 or $2 \mathrm{~h}$ prior to the designated retrieval time. 
Therefore, the final database consisted of sub-databases constructed at five different time steps before the selected retrieval period. The data and time of the radar and satellite data for each of the precipitation cases used in the construction of the a priori databases are shown in Table 1 . In addition, each sub-database was set to have a different weight based on the time difference from the designated retrieval. The weight was expressed as follows:

$T_{i}=\exp \left(\frac{-t_{i}}{t_{\max }}\right)$,

where $t_{i}$ is the interval between the construction time of the $i$ th sub-database and the designated retrieval time, and $t_{\max }$ is the largest time interval between the sub-databases and the retrieval. Since the radar and satellite data were available every 10 and $15 \mathrm{~min}$, respectively, there was a temporal mismatch of $5 \mathrm{~min}$ between the two data sets. In order to minimize the effects of the temporal inconsistency, the mismatched data set was assigned to have a smaller weight than the matched one.

As an example of the database characteristics, the relationships between the radar rain rate and the satellite $T_{\mathrm{b}}$ at the four different channels for the precipitation case 2 are shown in Fig. 3. Empty squares indicate a mean of the $T_{\mathrm{b}}$ of a $2 \mathrm{~mm} \mathrm{~h}^{-1}$ interval of the radar rain rate. It was observed that the $T_{\mathrm{b}}$ tended to decrease as the radar rain rate increased. Similar relationships for GOES- $8 T_{\mathrm{b}}$ at $10.7 \mu \mathrm{m}$ and the radar rain rate were also shown by Vicente et al. (1998).

\subsubsection{Retrievals and bias correction}

Once the a priori databases were constructed, the satellite precipitation estimation was achieved through the Bayesian inversion method as utilized by Shin and Kummerow (2003). In this approach, finding the posterior probability was the primary objective. The probability may be written as follows:

$P\left(R \mid T_{\mathrm{b}}\right)=P(R) \times P\left(T_{\mathrm{b}} \mid R\right)$,

where $P\left(R \mid T_{\mathrm{b}}\right)$ is the probability of a particular rain rate $R$ at a given $T_{\mathrm{b}}, P(R)$ is the probability that a specific rain rate can be observed, and $P\left(T_{\mathrm{b}} \mid R\right)$ is the probability of observing $T_{\mathrm{b}}$ at a particular $R$. As outlined by Rodgers (2000), the conditional probability $P\left(T_{\mathrm{b}} \mid R\right)$ may be simulated by a multi-dimensional Gaussian distribution of the difference between the observation and the corresponding simulation from a forward model. The uncertainty was also involved in the observation and forward simulation. However, in a retrieval using the observational database as in this study, the error from the forward computations would not appear in the covariance matrix for the term $P\left(T_{\mathrm{b}} \mid R\right)$. The covariance matrix has only the diagonal elements of the instrumental noises at each channel. Furthermore, since the $P(R)$ of the prior information, constructed during the period close to the designated retrieval time, was a good description of the true probability distribution of the precipitation fields observed by the

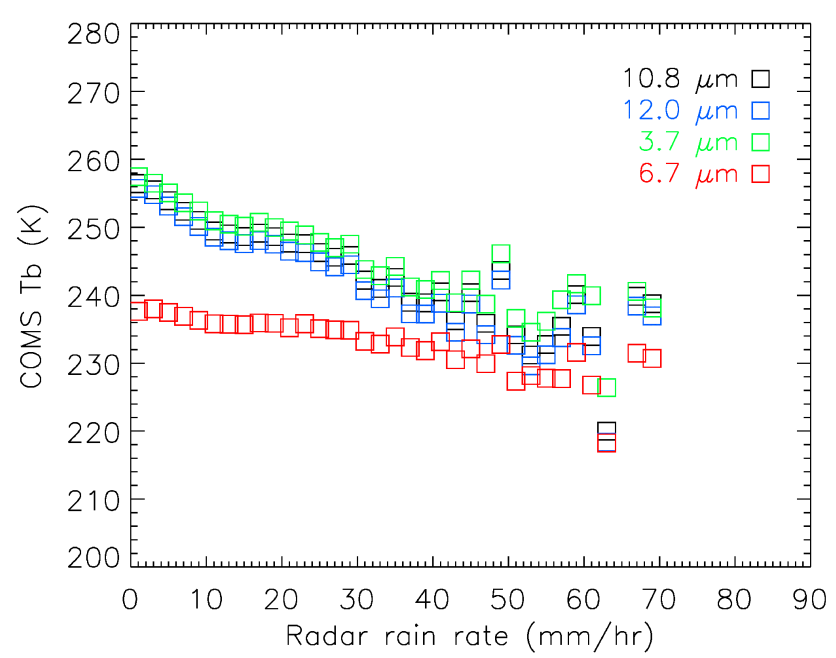

Figure 3. Mean relationship between the COMS brightness temperature $\left(T_{\mathrm{b}}\right)$ and the Jindo radar rain rate. Channels at 10.8, 12.0, 3.7 , and $6.7 \mu \mathrm{m}$ are indicated by black, blue, green, and red squares, respectively.

radar, the $P(R)$ was simply replaced by the frequency of each element in the prior information.

After the retrieval of the satellite precipitation, a bias correction based on an ensemble bias factor field (Tesfagiorgis et al., 2011b) computed from the radar precipitation was employed in order to improve the retrieval accuracy. The bias factor, which was defined as the ratio of a radar rain rate and the retrieved satellite precipitation at a specific location and time, was randomly selected over the study area. Ensembles of the perturbed bias factor and bias field were then generated. The details of the ensemble bias correction method were described by Tesfagiorgis et al. (2011b). By using the bias field, the bias-corrected precipitation estimation from the satellite was calculated. The radar-observed precipitation (left column) and the satellite-estimated precipitation with the bias correction (right column) from four of the precipitation cases are presented in Fig. 4. The spatial patterns of the satellite precipitations were relatively well matched to those of the radar observations. The bias-corrected satellite precipitation estimates were also compared to those by the radar observations in terms of the bias, root-mean-square error (RMSE), and correlation statistics (Fig. 5). The bias and correlation coefficients between the radar and satellite estimates ranged from 1.21 to $4.10 \mathrm{~mm} \mathrm{~h}^{-1}$ and from 0.46 to 0.61 , respectively. Although all of the estimation statistics tended to improve after the bias correction (not shown), a relatively large bias and RMSE still existed depending on the cases. The statistics might be attributed to the inherently indirect and inconsistent physical relationships between the surface rainfall and the satellite IR $T_{\mathrm{b}}$. 
Case 1
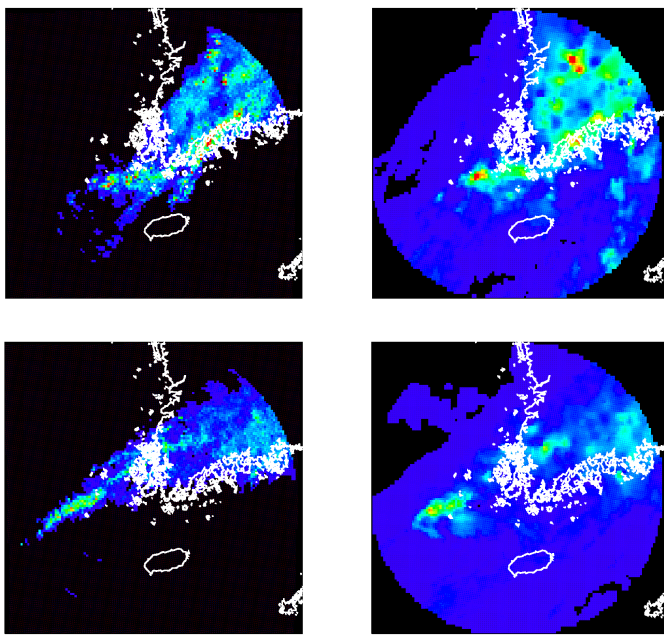

Case 2
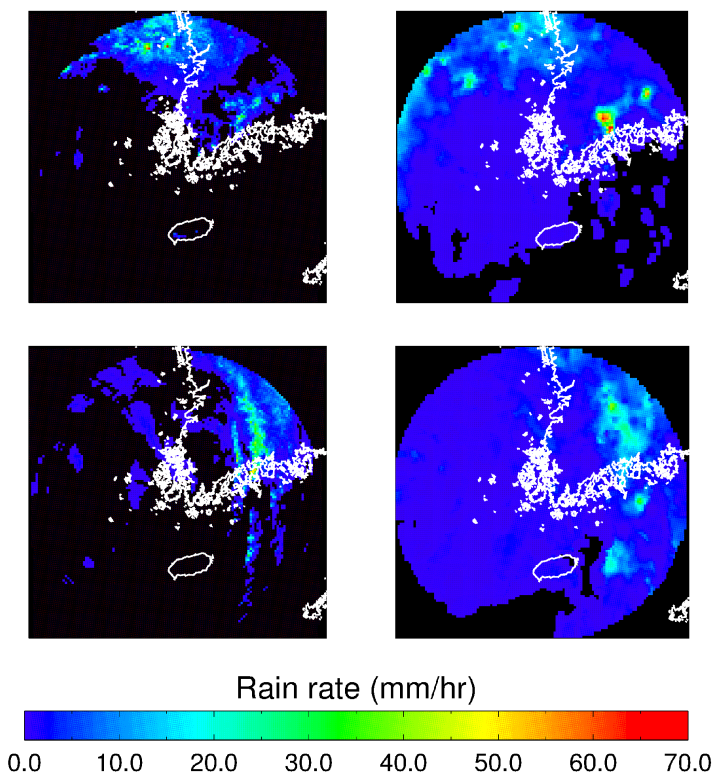

Figure 4. Images of the Jindo radar rain rates (left column) and satellite precipitation estimates after bias correction (right column) for the four precipitation cases.

\subsection{Merging radar and satellite precipitation estimates}

Precipitation estimation over radar gap areas was based on the merging of two precipitation fields: the bias-corrected satellite precipitation and the radar observations interpolated from the surrounding radar observations. The procedure to obtain the satellite precipitation estimates with bias correction was discussed in the previous section. The interpolation of the adjacent radar observations across the gap areas was performed using the following equation:
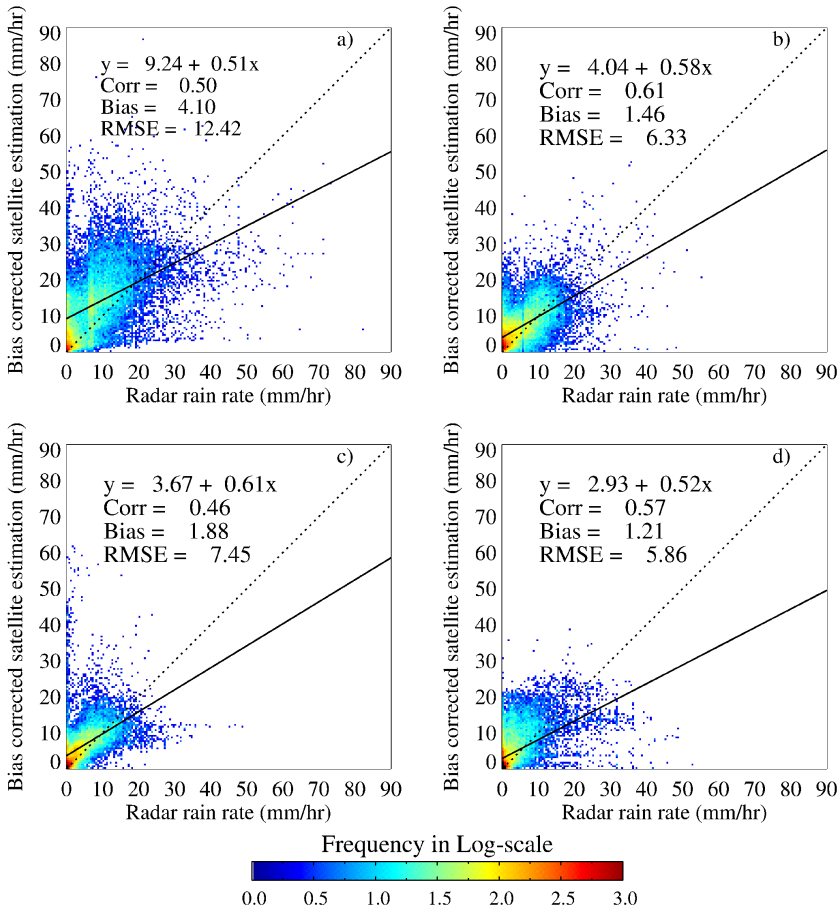

Figure 5. Scatter density diagrams of the radar rain rate estimation and satellite estimated precipitation after bias correction for the four precipitation cases of (a) case 1, (b) case 2, (c) case 3, and (d) case 4. Comparison statistics including correlation coefficient (Corr), bias, and root-mean-square error (RMSE) are also presented in each panel.

$R^{\prime}=\frac{\sum_{k=1}^{N} I_{k} \cdot R_{k}}{\sum_{k=1}^{N} I_{k}}$,

where $R^{\prime}$ is the interpolated radar rain rate at a pixel at the radar resolution over the gap area and $R_{k}$ is the radar observed rain rate at the $k$ th pixel among $N$ pixels within a $5 \mathrm{~km}$ distance from the location of $R^{\prime} . I_{k}$ is the $k$ th pixel's weight determined by the following Gaussian function:

$I_{k}=\exp \left(\frac{-d_{k}^{2}}{2 r^{2}}\right)$

where $d_{k}$ is the distance $(\mathrm{km})$ between the given pixel over the radar gap area and the $k$ th pixel over the surrounding area, and $r$ is the maximum distance $(\mathrm{km})$, which was set to $5 \mathrm{~km}$ in this study. We may then note that the interpolation is simply based on the distances between the pixel in the gap and the radar pixels in the surrounding area. The interpolation repeats at each pixel within the gap area. This interpolated value was merged with the matching satellite precipitation estimate at a pixel over the radar gap area. The method of 
merging was applied only within the gap area, so that the initial radar observations remained intact outside of the gap area.

In order to generate the merged precipitation field, the weight coefficients allocating the contribution from each data set over the radar gap area needed to be determined. The weight coefficients are calculated at each pixel. The proposed method in this study is thus considered a successive correction implying a pixel-by-pixel calculation of the gap (e.g., Brandes, 1975; Mahani and Khanbilvardi, 2009; Tesfagiorgis and Mahani, 2013). The previous study of Brandes (1975) applied a successive correction method to produce radar calibration fields using rain gauges. Mahani and Khanbilvardi (2009) and Tesfagiorgis and Mahani (2013) used satellite precipitation estimates and surrounding radar precipitation within a moving window. These studies utilized the difference between radar and satellite precipitation estimations of surrounding gap (outside of the gap) for generating merged precipitation. This study first estimates satellite precipitation and interpolates the radar rain rate field over the gap areas using surrounding radar-estimated precipitation. Optimal merging weights are then determined for the satellite precipitation and interpolated precipitation fields based on the RMSE difference from the reference data. The weight coefficient, $K_{l}$, for the $l$ th data set can be written as follows:

$$
K_{l}=\frac{\frac{1}{\sigma_{l}^{2}}}{\sum_{m=1}^{n} \frac{1}{\sigma_{m}^{2}}} \quad l=1, \ldots, n,
$$

where $\sigma_{l}$ and $\sigma_{m}$ are the RMSE difference from the reference data for the $l$ th and $m$ th data sets, respectively. In order to evaluate the accuracy of the merged precipitation field, the synthetic radar gap areas in the radar observational coverage were set and three experiments with the two different reference data sets and the equal weight were performed.

The first determined the optimal weights based on the original radar observation over the synthetic gap area. This experiment was not possible in practice, since the radar observations did not exist over the gap area. It was deliberately performed in order to compare with the other two merging experiments. The second experiment used the Automatic Weather Station (AWS) data as the reference observation. The AWS observation nearest to the merging pixel was selected in this experiment. The final one assigned equal weight coefficients to each data set $\left(K_{l}=1 / n\right)$. The weight coefficient was $1 / 2$ for the two data sets. This experiment could be used in case any reference data were not available over the gap area. The merging method works for either of the rain or no-rain cases over the gap areas. The results of the three experiments merging the interpolated radar precipitation field and bias-corrected satellite precipitation data over the radar gap area are presented in the next section. In the experiments, the radar gap areas were selected over significant raining regions with rain rates greater than $10 \mathrm{~mm} \mathrm{~h}^{-1}$ and its size was
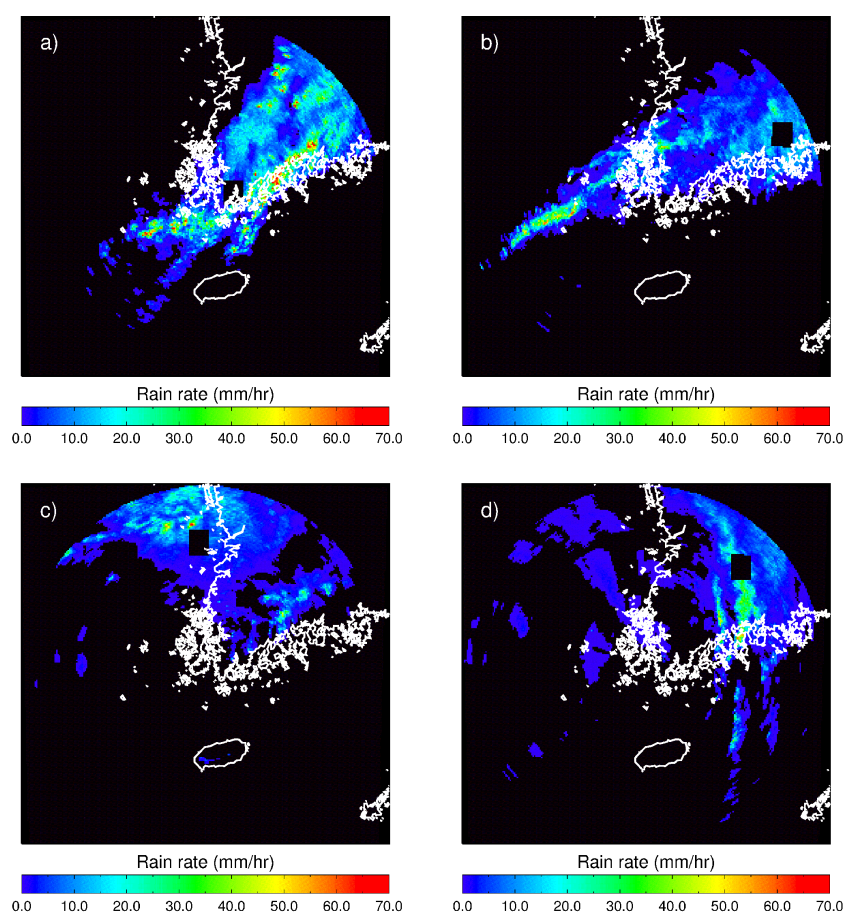

Figure 6. Images of the Jindo radar rain rate estimations for the four different precipitation cases of (a) case 1, (b) case 2, (c) case 3, and (d) case 4 . The black boxes in the rain images indicate the synthetic radar gap areas whose size is $0.3^{\circ} \times 0.3^{\circ}$ latitude-longitude.

set to $0.3^{\circ} \times 0.3^{\circ}$ latitude-longitude. The images for the four radar observation cases with the synthetically located gap areas (black box) are shown in Fig. 6.

\section{Result and discussions}

Precipitation estimations over the gap areas by the three experiments were performed for the four different precipitation cases. All of the selected gap areas were filled with rainfalls. The results are presented in Fig. 7. Again, the three experiments merging the bias-corrected satellite and interpolated radar precipitation fields using the radar-determined weights, the AWS-driven weights, and the equal weights without the reference data are shown in the columns of Exp. 1, Exp. 2, and Exp. 3, respectively. All three of the experiments roughly displayed patterns similar to those in the original images of the radar (the first column) with a certain degree of discontinuity around the boundaries of the gap areas. In particular, discontinuity was found in the second and third experiments that adopted the merging approaches based on the AWS data and equal weights (columns of Exps. 2 and 3). The optimal weight merging method using the radar as a reference sensor seemed to simulate the precipitation field with the smallest discontinuity around the gap areas in all of the precipitation cases (column of Exp. 1). 


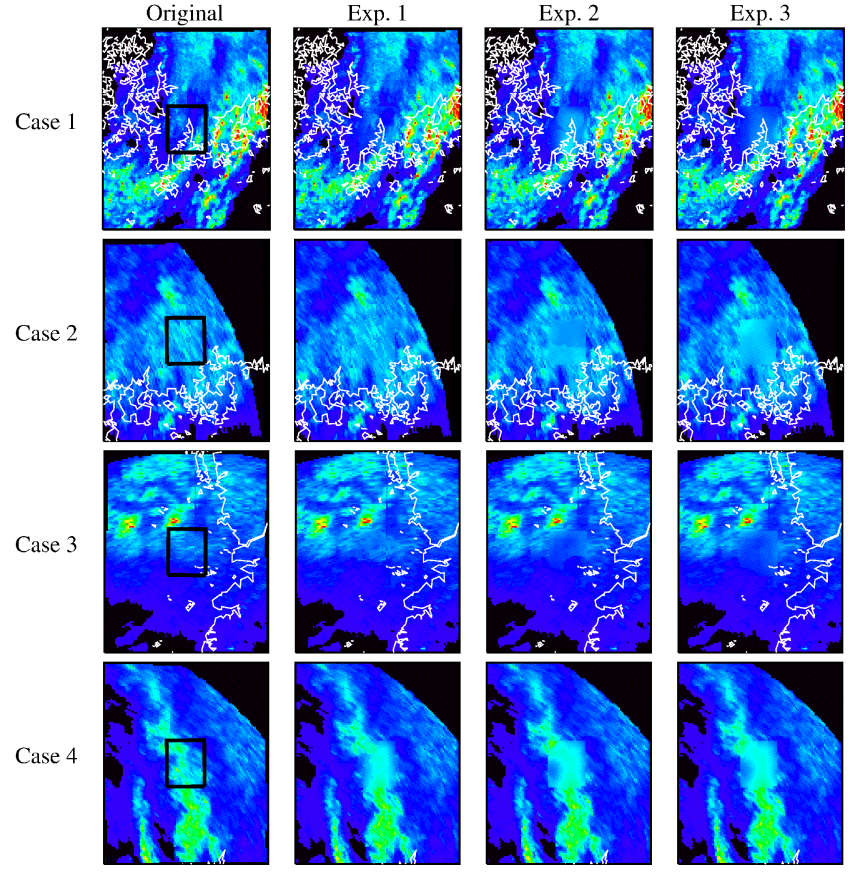

Rain rate $(\mathrm{mm} / \mathrm{hr})$

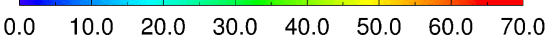

Figure 7. Precipitation distributions estimated by the Jindo radar for the four precipitation cases (the first column), same as in Fig. 6, but focused on the surrounding of the gap areas (black line box). Precipitation estimations over the black boxes from the three merging experiments employing the optimal weights determined by the original radar data and the AWS data, and the equal weights $(1 / 2)$ are illustrated in the rest of the columns, respectively.

Figure 8 also compares the accuracy of the three merging experiments for each of the precipitation cases. The first experiment (column Exp. 1) appeared to produce higher quality precipitation estimates over the gap areas with high correlations $(0.71,0.81,0.90$, and 0.66$)$ and relatively low biases $(-0.50,0.23,-0.56$, and 1.41$)$ for the four precipitation cases. This result may have been expected, because the original radar observations over the gap areas were involved in the merging process. On the other hand, the other merging methods (Exps. 2 and 3) did not appear to produce comparable results relative to the first experiment. In addition, the varying estimation statistics may suggest that the accuracy of the merged results tended to depend on the precipitation cases and the location of the gap areas. In particular, the correlation coefficients from the second experiment based on the AWS data turned out to be highly variable ranging from -0.06 to 0.76 .

Comparisons of the radar observations, satellite estimates, and merged results for their mean and standard deviations are also presented in Table 2. In this study, the relative difference was the ratio of the difference between the mean satellite estimate (or the mean merged estimate) and the mean radar rain rate to the mean radar rain rate. The standard deviations of the merged results were less than those of the radar observations for all of the precipitation, implying that the variability of the merged precipitation field was smaller than that of the radar observation. For example, as shown in Table 2, the relative differences for the three merging experiments were quite small $\left(0.02,-0.01\right.$, and $\left.0.10 \mathrm{~mm} \mathrm{~h}^{-1}\right)$ for the precipitation case 2, while the standard deviation differences between the radar and merged precipitations were still noticeable. This result suggests that the smaller variability appeared to be a characteristic of the merged precipitation field and made a distinct discontinuity around the gap areas in case 2 (Fig. 7), regardless of the small relative differences. The other highlight was that the absolute relative differences of the merged results were smaller than those of the satellite estimates in most of the cases. This indicates that the merging methods tended to lead to better agreement with the radar observation than applying only the satellite precipitation estimates to the radar gap areas.

An attempt was made to analyze the impacts of the satellite precipitation estimates on the merged results with additional radar gap areas at different locations and two different precipitation cases including 10 July 2011, 12:00 (local time) and 7 August 2011, 16:00. Figure 9 shows the scatter diagrams of two of the correlation coefficients. The first correlation $\left(\rho_{\mathrm{sr}}\right)$ was obtained from the satellite estimates and the radar observations. The second $\left(\rho_{\mathrm{mr}}\right)$ indicated the relationship between the merged precipitation estimates and the radar observation. Each of the scatter diagrams represents one of the three merging methods: (a) optimal weight using radar, (b) optimal weight using AWS, and (c) equal weight. An increasing trend of $\rho_{\mathrm{mr}}$ with an increase in $\rho_{\text {sr }}$ existed, indicating that highly correlated satellite precipitation estimates with radar observations produced well matched merged precipitation fields over the radar gap with radar observation, especially with merging methods of optimal weight using radar and equal weight. In other words, more accurate precipitation estimation from satellites created better merged results. This indicates that the accuracy of the merged results depended on the accuracy of the satellite precipitation estimates. It was also obvious that $\rho_{\mathrm{mr}}$ was typically larger than $\rho_{\text {sr }}$ except for a few cases in the merging with AWS and equal weight. This result implies that using merged precipitation fields helped to estimate more accurate precipitation than using only satellite precipitation estimation over the radar gap area. In addition, the optimal weight merging using the AWS method showed less dependence on satellite estimates. This result may have been due to the sparse distribution of the AWS observation data, which failed to resemble the spatial characteristics of the radar observation.

The performance of the three merging methods was tested (Fig. 10). The method of optimal weight merging using radar clearly showed better performance than the other methods. The other notable feature was that the equal weight 
a) Exp. 1

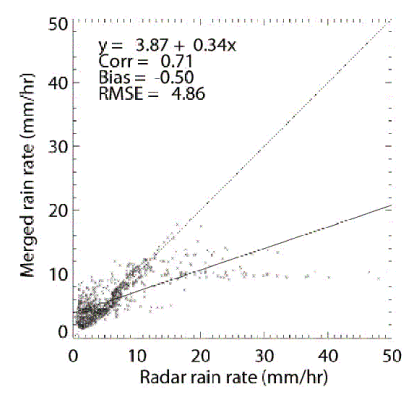

Case 2
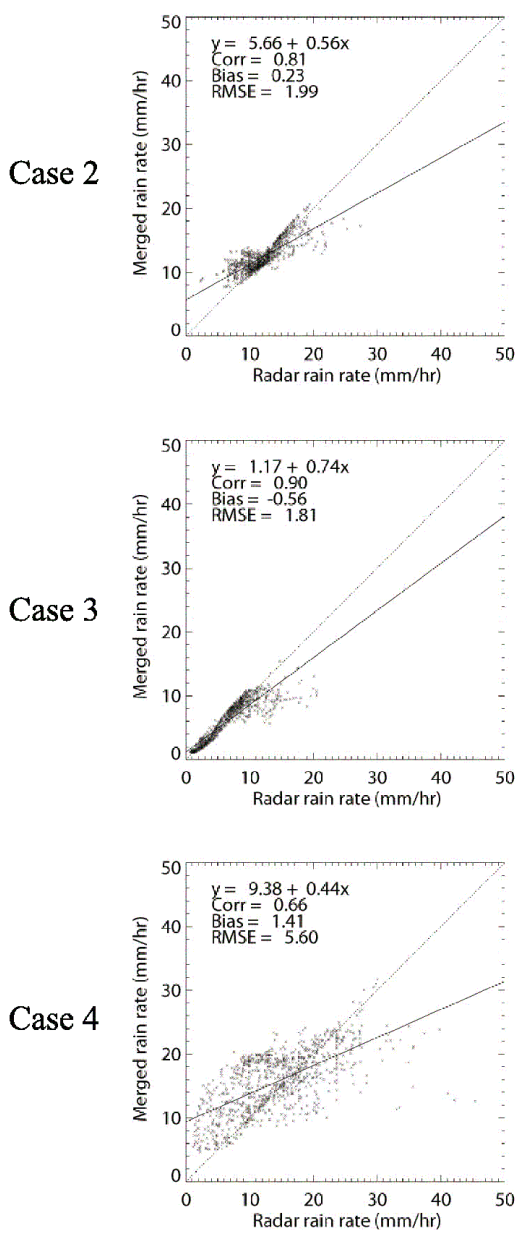

b) Exp. 2
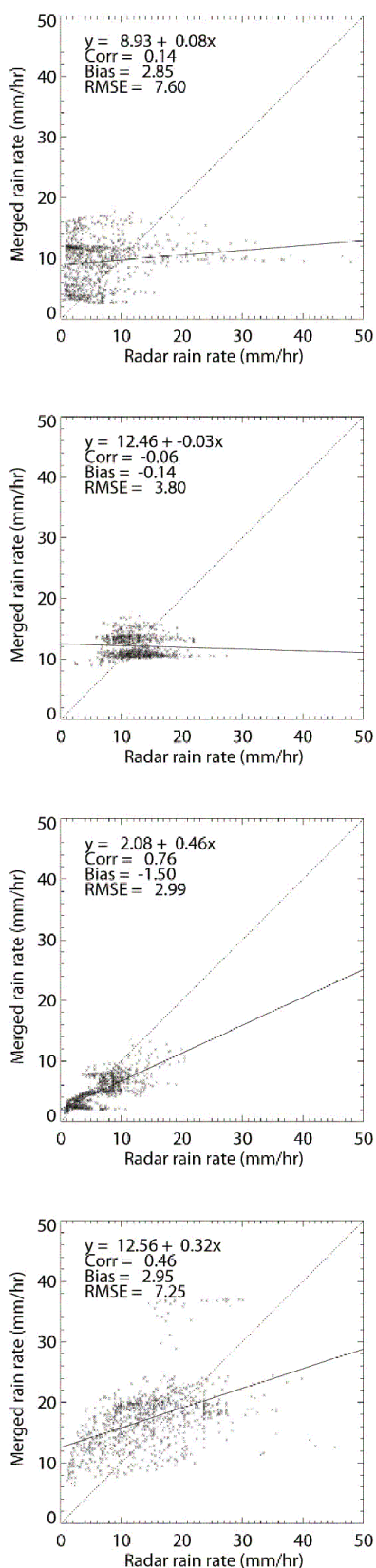

c) Exp. 3
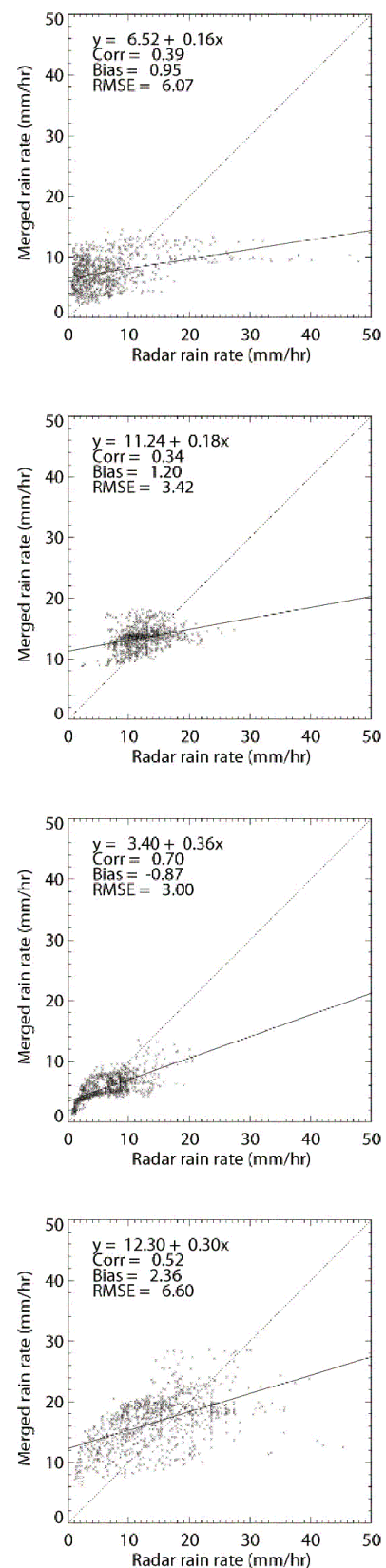

Figure 8. Scatter diagrams for the radar rain rate estimations vs. the merged precipitation over the radar gap areas using the merging methods of (a) optimal weight using radar, (b) optimal weight using AWS, and (c) equal weight for each of the precipitation cases.

merging method produced better merged results than the optimal weight merging using AWS. This result suggests that the optimal weight information from the AWS appeared to be inaccurate. The reason for this inaccuracy, as we have discussed previously, may be that the distance between the gap pixel and the AWS (maximum $5 \mathrm{~km}$ ) did not guarantee an accurate description of the spatial characteristics of the radar observation.

\section{Summary}

The merging methods of radar and satellite precipitation estimates over radar gap areas were presented in this study. This method first retrieved satellite-based precipitation using a priori databases constructed from the radar at the Jindo site $\left(126.3^{\circ} \mathrm{E}, 34.5^{\circ} \mathrm{N}\right)$ and the COMS-observed $T_{\mathrm{b}}$ at each IR channel. Retrieval of precipitation was achieved through a Bayesian inversion, which depended on the 
Table 2. Mean $\left(\mathrm{mm} \mathrm{h}^{-1}\right)$ and standard deviation $(\mathrm{SD})\left(\mathrm{mm} \mathrm{h}^{-1}\right)$ values of the radar observation, satellite precipitation estimates after bias correction, and radar-satellite merged results over the radar gap areas for the four precipitation cases. In this study, the relative difference indicates the ratio of the difference between the satellite estimates or merged results from the radar observation to the mean radar observation over the radar gap area.

\begin{tabular}{|c|c|c|c|c|c|c|c|c|c|c|c|c|c|c|}
\hline \multirow{3}{*}{ Precipitation case } & \multirow{2}{*}{\multicolumn{2}{|c|}{ Radar }} & \multirow{2}{*}{\multicolumn{3}{|c|}{ Satellite estimates }} & \multicolumn{9}{|c|}{ Merged results } \\
\hline & & & & & & \multicolumn{3}{|c|}{$\begin{array}{c}\text { Optimal weight } \\
\text { merging - radar (Exp. 1) }\end{array}$} & \multicolumn{3}{|c|}{$\begin{array}{c}\text { Optimal weight } \\
\text { merging - AWS (Exp. 2) }\end{array}$} & \multicolumn{3}{|c|}{$\begin{array}{l}\text { Equal weight } \\
\text { merging (Exp. 3) }\end{array}$} \\
\hline & Mean & SD & Mean & SD & $\begin{array}{r}\text { Relative } \\
\text { difference }\end{array}$ & Mean & SD & $\begin{array}{l}\text { Relative } \\
\text { difference }\end{array}$ & Mean & SD & $\begin{array}{l}\text { Relative } \\
\text { difference }\end{array}$ & Mean & $\mathrm{SD}$ & $\begin{array}{r}\text { Relative } \\
\text { difference }\end{array}$ \\
\hline 1 & 6.61 & 6.51 & 5.04 & 3.14 & -0.24 & 6.10 & 3.11 & -0.08 & 9.45 & 3.74 & 0.43 & 7.56 & 2.64 & 0.14 \\
\hline 2 & 12.26 & 3.33 & 16.12 & 2.97 & 0.31 & 12.49 & 2.28 & 0.02 & 12.12 & 1.65 & -0.01 & 13.46 & 1.79 & 0.10 \\
\hline 3 & 6.63 & 3.88 & 8.33 & 2.24 & 0.26 & 6.07 & 3.20 & -0.08 & 5.14 & 2.34 & -0.22 & 5.76 & 1.97 & -0.13 \\
\hline 4 & 14.24 & 7.20 & 18.48 & 5.43 & 0.30 & 15.65 & 4.82 & 0.10 & 17.19 & 5.08 & 0.21 & 16.60 & 4.19 & 0.17 \\
\hline
\end{tabular}
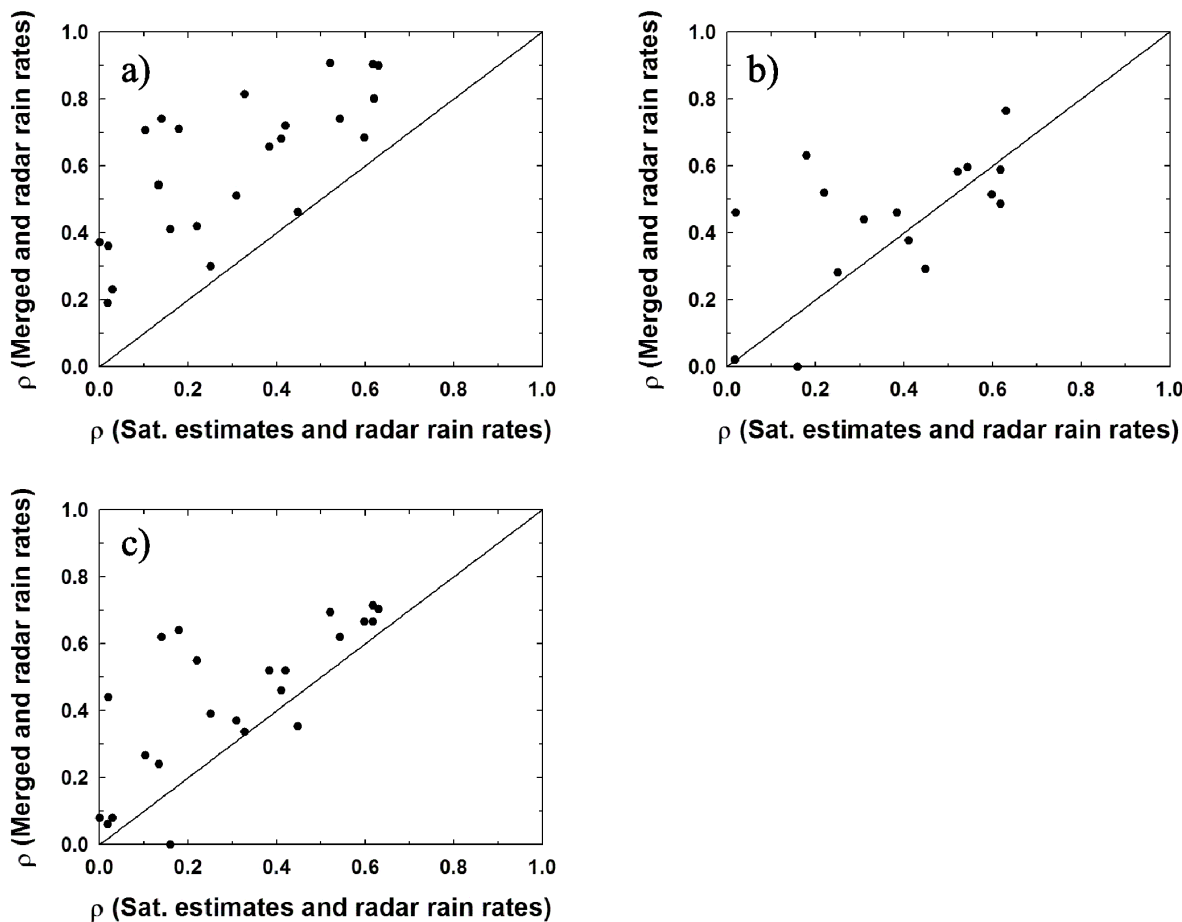

Figure 9. Scatter diagrams of the two correlation coefficients. The first correlation in the abscissa is obtained from the satellite estimates and radar rain rate estimations. The other correlation in the ordinate is from the merged precipitation estimates and the radar rain rate estimations. Panels are classified by the three merged precipitation estimates from (a) optimal weight using radar, (b) optimal weight using AWS, and (c) equal weight.

relationships between the radar rain rate and the satellite $T_{\mathrm{b}}$ in the database. The initial satellite estimates were then adjusted by an ensemble bias correction method (Tesfagiorgis et al., 2011b) using the radar data.

The merging of the bias-corrected satellite precipitation and interpolated radar precipitation fields over the radar gap areas was implemented by optimal weight merging. The weights were calculated optimally from the radar and AWS. For comparison, an additional merging experiment with equal weight was included.
The results showed that the optimal weight merging method using radar data produced the minimum discontinuity around the radar gap areas, which were synthetically defined to test the merging methods (Fig. 7). The accuracy of the merged results was evaluated by comparing the merged results and the original radar observations over the radar gap area. The optimal weight using the radar method outperformed the other merging methods based on the AWS and equal weights with relatively high correlation coefficients (0.66-0.90). The equal weight merging method without any reference information generated merged precipitation over 


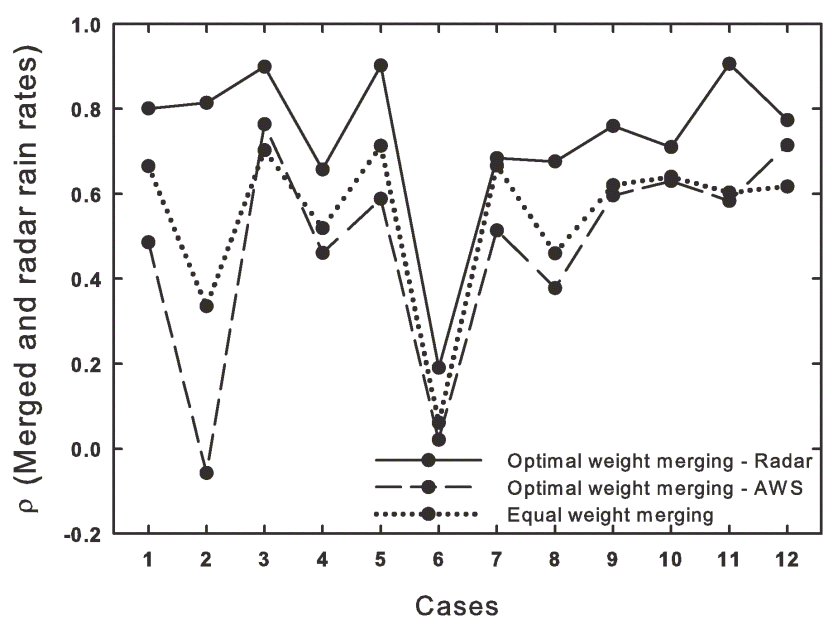

Figure 10. The correlation coefficients between the merged precipitation and radar rain rate estimation over the different gap areas. The merged precipitation estimates are obtained from the merging methods of optimal weight using radar (solid line), optimal weight using AWS (dashed line), and equal weight (dotted line).

the radar gap area, with variable correlation coefficients of 0.34-0.70 depending on the precipitation cases (Fig. 8). The results of this experiment may be used to produce practical precipitation estimates when any other ground observation is not available. In terms of the mean value of precipitation over the gap areas, the merged precipitation field agreed with the original radar data within $\sim 17 \%$ except for one method of merging (Table 2). More importantly, the results of this study demonstrate that more accurate precipitation estimation from satellites is essential in order to create better merging results over the radar gap areas (Fig. 9).

Acknowledgements. This research was supported by the "Development and application of cross governmental dual-pol radar harmonization (WRC-2013-A-1)" project of the Weather Radar Center, Korea Meteorological Administration in 2014.

Edited by: M. Portabella

\section{References}

Austin, P. M.: Relation between measured radar reflectivity and surface rainfall, Mon. Weather Rev., 115, 1053-1070, 1987.

Brandes, E. A.: Optimizing rainfall estimates with the aid of radar, J. Appl. Meteor., 14, 1339-1345, 1975.

Choi, Y.-S., Ho, C.-H., Ahn, M.-H., and Kim, Y.-M.: An exploratory study of cloud remote sensing capabilities of the Communication, Ocean and Meteorological Satellite (COMS) imagery, Int. J. Remote Sens., 28, 4715-4732, 2007.

Kidd, C., Kniveton, D. R., Todd, M. C., and Bellerby, T. J.: Satellite rainfall estimation using combined passive microwave and infrared algorithms, J. Hydrometeor., 4, 1088-1104, 2003.

Kondragunta, C., Kitzmiller, D., Seo, D. J., and Shrestha, K.: Objective integration of satellite, rain gauge, and radar precipitation estimates in the multisensor precipitation estimator algorithm, 19th Conference on Hydrology, San Diego, CA, Amer. Meteor. Soc., 2005.

Mahani, S. E. and Khanbilvardi, R.: Generating multi-sensor precipitation estimates over radar gap areas, WSEAS Transactions on Systems, 8, 96-106, 2009.

Rodgers, C. D.: Inverse Methods for Atmospheric Sounding: Theory and Practice, World Scientific, Singapore, 2000.

Scofield, R. A. and Kuligowski, R. J.: Status and outlook of operational satellite precipitation algorithms for extreme-precipitation events, Weather Forecast., 18, 1037-1051, 2003.

Shin, D.-B. and Kummerow, C.: Parametric rainfall retrieval algorithms for passive microwave radiometers, J. Appl. Meteorol., 42, 1480-1496, 2003.

Tesfagiorgis, K. and Mahani, S. E.: A multi-source precipitation estimation approach to fill gaps over a radar precipitation field: a case study in the Colorado River Basin, Hydrol. Process., 29, 29-42, doi:10.1002/hyp.10103, 2013.

Tesfagiorgis, K., Mahani, S. E., and Khanbilvardi, R.: Multi-sources precipitation estimation: Mitigating gaps over radar network coverage, in: Proceedings of IGARSS, Vancouver, BC, 3054-3056, 2011a.

Tesfagiorgis, K., Mahani, S. E., Krakauer, N. Y., and Khanbilvardi, R.: Bias correction of satellite rainfall estimates using a radar-gauge product - a case study in Oklahoma (USA), Hydrol. Earth Syst. Sci., 15, 2631-2647, doi:10.5194/hess-15-26312011, 2011b.

Vicente, G. A., Scofield, R. A., and Menzel, W. P.: The operational GOES infrared rainfall estimation technique, B. Am. Meteor. Soc., 79, 1883-1898, 1998. 